\title{
DRD2 haplotype associated with negative symptoms and sustained attention deficits in Han Chinese with schizophrenia in Taiwan
}

\author{
Yi-Ling Chien ${ }^{1}$, Hai-Gwo Hwu ${ }^{1,2,3,4}$, Cathy S-J Fann ${ }^{5}$, Chien-Ching Chang ${ }^{5}$, Ming-T Tsuang ${ }^{6,7,8}$ \\ and Chih-Min Liu ${ }^{1,2}$
}

Previous studies have reported significant associations between schizophrenia and the dopamine receptor D2 gene (DRD2) variants. The relationships between DRD2 and clinical phenotypes are of particular interest because DRD2 has been shown to associate with treatment response and prefrontal dopamine transmission. Glatt et al. reported significant associations between schizophrenia and DRD2 variants (two single-nucleotide polymorphisms (SNPs) rs1079727 and rs2283265, and two haplotypes, block 3 (rs1079727(A)-rs2440390(C)-rs2283265(G)) and block 4 (rs1801028(G)-rs1110977(A)-

rs1124492(C)-rs2734841 (T))) in 2408 Han Chinese individuals in Taiwan. To further investigate the relationships between the SNPs/haplotypes of DRD2 and clinical symptoms and neuropsychological function, we compared the quantitative phenotypes in patients with risk alleles/haplotypes and those without. The results showed that the A allele of rs1079727, $G$ allele of rs2283265, A allele of rs 1124492 and the risk haplotype (A-C-G) of block 3 were associated with more severe negative symptoms. With regard to neuropsychological performance, the risk haplotype (G-A-C-T) of block 4 was associated with poorer performance in the sustained attention task. Our results imply that the genetic variants of DRD2 might not only have a role in susceptibility to schizophrenia, but also influence the phenotypes of negative symptoms and sustained attention in schizophrenia. This association warrants further validation.

Journal of Human Genetics (2013) 58, 229-232; doi:10.1038/jhg.2012.157; published online 31 January 2013

Keywords: dopamine receptor D2 gene (DRD2); haplotype; neuropsychological function; schizophrenia

The dopamine receptor D2 gene (DRD2) has been shown by numerous meta-analyses to contribute to the susceptibility of individuals for schizophrenia across populations. ${ }^{1-3}$ Glatt et al. ${ }^{4}$ used a family-based association study to test 21 haplotypetagging and all three known non-synonymous single-nucleotide polymorphisms (SNPs) in DRD2 for association with schizophrenia in 2408 Han Chinese individuals in Taiwan, including 1214 patients from 616 families with at least two affected siblings. The results showed a significant association with two haplotypes (block 3, composed of rs1079727(A)-rs2440390(C)-rs2283265(G), $P=0.0047$ and block 4, rs1801028(G)-rs1110977(A)-rs1124492(C)rs2734841(T), $P=0.0023)$ of DRD2 after multiple testing corrections. Two SNPs in block 3, rs1079727 $(P=0.0041)$ and rs2283265 $(P=0.0051)$, were also significantly associated. The relationships between these SNPs/haplotypes and clinical symptoms and neuropsychological function are of particular interest because
DRD2 has been shown to be associated with treatment response ${ }^{5}$ and prefrontal dopamine transmission. ${ }^{6}$

To further investigate the relationships between these SNPs/ haplotypes and clinical symptoms and neuropsychological function, we compared the quantitative phenotypes between patients with risk alleles/haplotypes and those without risk alleles/haplotypes for the above seven SNPs and two blocks, in the same samples used in Glatt et al.'s study. ${ }^{4}$ The sample characteristics and the methodology of DNA acquisition, marker selection and genotyping were described in detail by Hwu et al. ${ }^{7}$ and Glatt et al. ${ }^{4}$ The genetic association was tested by using a generalized estimated equations model with an exchangeable correlation structure. The Bonferroni correction was performed for multiple comparisons. The quantitative phenotypes included clinical symptoms using the schedule for assessment of negative symptoms and the schedule for assessment of positive symptoms, and two neuropsychological tests (the continuous

\footnotetext{
${ }^{1}$ Department of Psychiatry, National Taiwan University Hospital, Taipei, Taiwan; ${ }^{2}$ Department of Psychiatry, National Taiwan University College of Medicine, Taipei, Taiwan; ${ }^{3}$ Institute of Epidemiology, College of Public Health, National Taiwan University, Taipei, Taiwan; ${ }^{4}$ Department of Psychology, College of Science, National Taiwan University, Taipei, Taiwan; ${ }^{5}$ Institute of Biomedical Sciences, Academia Sinica, Taipei, Taiwan; ${ }^{6}$ Department of Psychiatry, Center for Behavioral Genomics, University of California, San Diego, La Jolla, CA, USA; ${ }^{7}$ Harvard Departments of Epidemiology and Psychiatry, Harvard Institute of Psychiatric Epidemiology and Genetics, Boston, MA, USA and ${ }^{8}$ Veterans Affairs San Diego Healthcare System, San Diego, CA, USA

Correspondence: Dr C-M Liu, Department of Psychiatry, National Taiwan University Hospital, No.7, Chung San South Road, Taipei, Taiwan. E-mail: cmliu1968@ntu.edu.tw
}

Received 25 July 2012; revised 15 November 2012; accepted 18 December 2012; published online 31 January 2013 


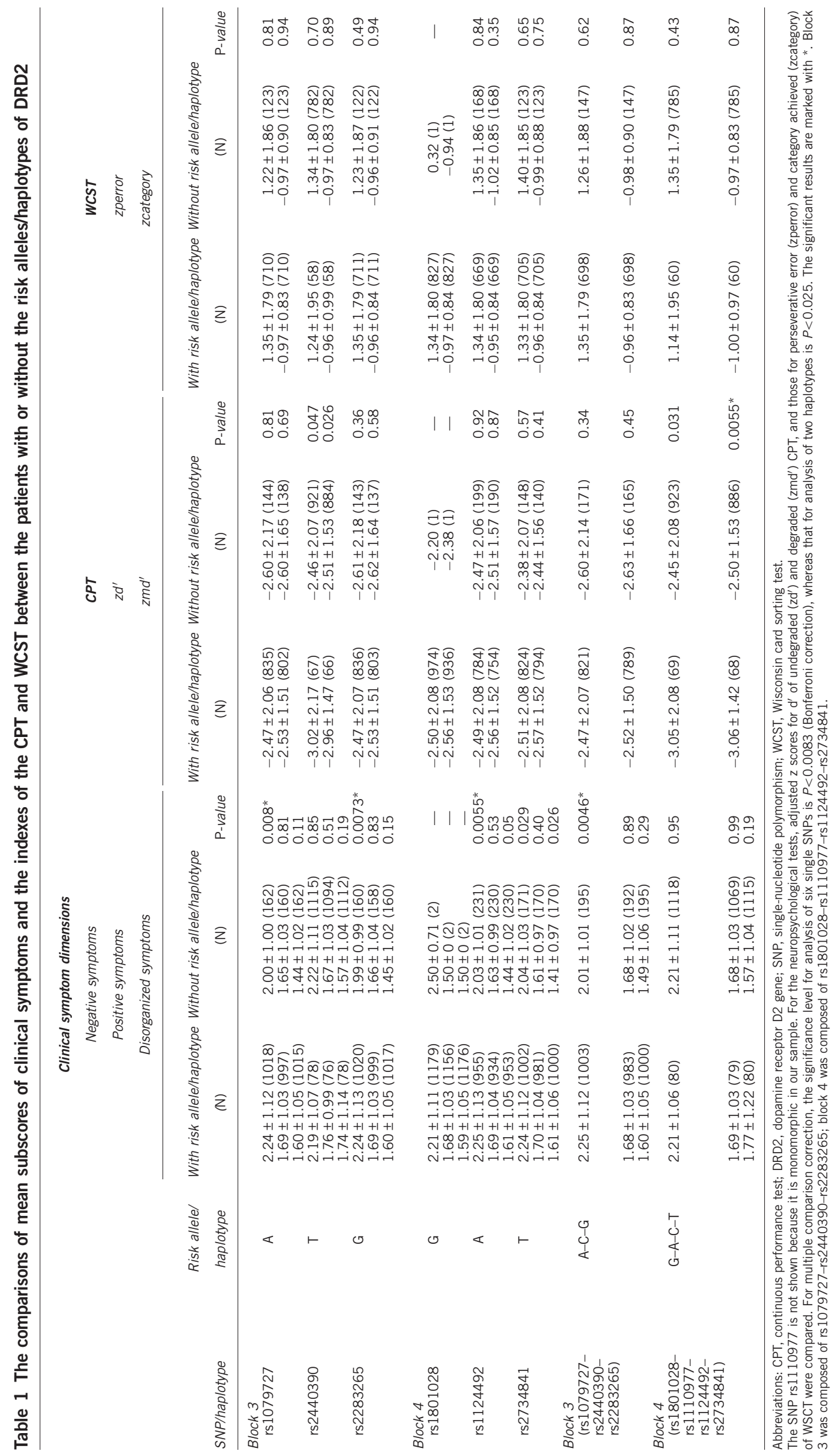


performance test (CPT, $n=992)$ and the Wisconsin card sorting test (WCST, $n=845$ )), which are widely used as valid tools to measure sustained attention and executive function, respectively. Deficits on the CPT and WCST have been proposed to be endophenotypes for schizophrenia, ${ }^{8-11}$ for that not only the patients themselves, but their unaffected relatives performed worse on these two tasks.

As to the schedule for assessment of negative symptoms and schedule for assessment of positive symptoms, the mean scores of positive symptoms, negative symptoms and disorganizing symptoms were compared. The mean scores were the mean of all global scores for the four negative symptom dimensions (affective blunting, alogia, avolition-apathy, and anhedonia-asociality), positive symptom dimensions (hallucination and delusion) and disorganizing symptom dimensions (bizarre behavior and positive formal thought disorder), respectively. For the neuropsychological tests, adjusted $\mathrm{z}$ scores for the $\mathrm{d}^{\prime}$ of undegraded $\left(\mathrm{zd}^{\prime}\right)$ and degraded CPT $\left(\mathrm{zmd}^{\prime}\right)$, and those for perseverative error (zperror) and category achieved (zcategory) on the WSCT were compared.

The single point association analysis revealed that the patients carrying the A allele of rs1079727, G allele of rs2283265 and A allele of rs1124492 had significantly higher mean negative symptom scores (corrected $P<0.05$; Table 1). However, there were no significant differences in the mean scores of positive symptoms and disorganizing symptoms or in CPT and WCST indexes if one carried a risk SNP. For haplotype analysis, patients carrying a risk haplotype (A-C-G) of block 3 had a significantly higher mean negative symptom score, whereas there were no significant differences in positive symptoms and disorganizing symptoms (Table 1). As to neuropsychological performance, patients carrying a risk haplotype (G-A-C-T) of block 4 had a lower $\mathrm{d}^{\prime}$ of degraded CPT and a trend toward lower undegraded CPT, whereas no significant difference was found in WCST performance (Table 1).

Previous studies regarding DRD2 and clinical symptoms mostly focused on Ser311Cys (rs1801028). ${ }^{12}$ This SNP was reported to associate with schizophrenia by meta-analysis ${ }^{1-3}$ and has been suggested to be associated with fewer negative symptoms and less thought disorder, early onset and family history of schizophrenia, ${ }^{13,14}$ but it also has been associated with disorganization ${ }^{15}$ and delusional symptoms across major psychoses. ${ }^{16}$ In our study, most of the subjects carried the risk allele for Ser311Cys, which rendered comparisons of clinical symptoms and neuropsychological performance between those with and without the risk allele inadequate to reach a conclusion. However, we found a significant association between a nearby risk SNP, rs1124492, in linkage disequilibrium with rs1801028, and negative symptoms. We also found that two SNPs in block 3 (rs1079727, rs2283265) and the A-CG haplotype of block 3 were significantly associated with higher negative symptom scores, which has not been reported previously.

The SNP rs1799732 in the promoter region has been reported to be associated with WCST performance in female patients with schizophrenia. ${ }^{17}$ We found no association between WCST and the seven SNPs and two blocks of the DRD2 gene, although these did not cover the promoter region, in our whole sample and our female subsample. Therefore, we cannot directly replicate the previous findings. The SNP rs6277 (Xu et al. ${ }^{18}$ ) and a haplotype of rs1800497-rs6277-rs2283265 (Markett et al. ${ }^{19}$ ) involved in working memory in healthy subjects have been measured by the word serial position test ${ }^{18}$ or a brief visual array test. ${ }^{19}$ The SNPs rs6277 and rs2283265 are located in block 4 and block 3, respectively, in our linkage disequilibrium map. Yet, we found a significant association between a risk haplotype of block 4 and sustained attention as measured by the CPT. The SNP rs6277 has also been reported to increase striatal DRD2 binding in humans ${ }^{20}$ and to be associated with schizophrenia. ${ }^{21,22}$ Taken together, ours with previous findings indicate that block 3 and block 4 of DRD2 may have a more important role in working memory and sustained attention in both healthy subjects and patients with schizophrenia. Our study provides more evidence for the involvement of DRD2 in sustained attention and working memory.

Several limitations of this study should be discussed. First, the samples were recruited mostly from the outpatient clinic and were not in an active phase of disease, which might mask the association with positive symptoms. However, as negative symptoms are relatively stable in chronic schizophrenia, the association with negative symptoms seemed more robust. Second, the drug effect on the performance of the neuropsychological task was not controlled. Third, the uneven sample sizes between the group with risk allele and the group without in some of the analyses may limit us in reaching a definite conclusion, particularly in analysis involving the SNP rs1801028. Nevertheless, the significant associations of rs1079727, rs2283265, rs1124492, block 3 and block 4 of DRD2 imply that the genetic variants of DRD2 may not only have a role in the susceptibility to schizophrenia as implicated by meta-analyses, but also influence the phenotypes of negative symptoms and sustained attention in schizophrenia. This association warrants validation from other independent samples.

\section{CONFLICT OF INTEREST}

The authors declare no conflict of interest.

\section{ACKNOWLEDGEMENTS}

This work was supported by grants from the National Institute of Mental Health, (IRO1 MH59624-01) and the National Science Council (NSC 96-3112B-002-011, NSC97-3112-B-002-046) in Taiwan.

1 Glatt, S. J. \& Jonsson, E. G. The Cys allele of the DRD2 Ser311Cys polymorphism has a dominant effect on risk for schizophrenia: evidence from fixed- and random-effects meta-analyses. Am. J. Med. Genet. B Neuropsychiatr. Genet. 141B, 149-154 (2006).

2 Allen, N. C., Bagade, S., McQueen, M. B., Ioannidis, J. P., Kavvoura, F. K., Khoury, M. J. et al. Systematic meta-analyses and field synopsis of genetic association studies in schizophrenia: the SzGene database. Nat. Genet. 40, 827-834 (2008).

3 Liu, Z. W., Liu, J. L., An, Y., Zhang, L. \& Wang, Y. M. Association between Ser311Cys polymorphism in the dopamine D2 receptor gene and schizophrenia risk: a metaanalysis in Asian populations. Genet. Mol. Res. 11, 261-270 (2012).

4 Glatt, S. J., Faraone, S. V., Lasky-Su, J. A., Kanazawa, T., Hwu, H. G. \& Tsuang, M. T. Family-based association testing strongly implicates DRD2 as a risk gene for schizophrenia in Han Chinese from Taiwan. Mol. Psychiatry. 14, 885-893 (2009).

5 Zhang, J. P., Lencz, T. \& Malhotra, A. K. D2 receptor genetic variation and clinical response to antipsychotic drug treatment: a meta-analysis. Am. J. Psychiatry 167, 763-772 (2010).

6 Wang, M., Vijayraghavan, S. \& Goldman-Rakic, P. S. Selective D2 receptor actions on the functional circuitry of working memory. Science 303, 853-856 (2004).

7 Hwu, H. G., Faraone, S. V., Liu, C. M., Chen, W. J., Liu, S. K., Shieh, M. H. et al. Taiwan schizophrenia linkage study: the field study. Am. J. Med. Genet. B Neuropsychiatr. Genet. 134B, 30-36 (2005).

8 Chen, W. J., Chang, C. H., Liu, S. K., Hwang, T. J. \& Hwu, H. G. Sustained attention deficits in nonpsychotic relatives of schizophrenic patients: a recurrence risk ratio analysis. Biol. Psychiatry 55, 995-1000 (2004).

9 Chen, W. J. \& Faraone, S. V. Sustained attention deficits as markers of genetic susceptibility to schizophrenia. Am. J. Med. Genet. 97, 52-57 (2000).

10 Wang, Q., Chan, R., Sun, J., Yao, J., Deng, W., Sun, X. et al. Reaction time of the continuous performance test is an endophenotypic marker for schizophrenia: a study of first-episode neuroleptic-naive schizophrenia, their non-psychotic first-degree relatives and healthy population controls. Schizophr. Res. 89, 293-298 (2007).

11 Ma, X., Wang, Q. Sham, P. C. Liu, X, Rabe-Hesketh, S., Sun, X et al. Neurocognitive deficits in first-episode schizophrenic patients and their first-degree relatives. Am. J. Med. Genet. B Neuropsychiatr. Genet. 144B, 407-416 (2007).

12 Sasaki, T., Macciardi, F. M., Badri, F., Verga, M., Meltzer, H. Y., Lieberman, J. et al. No evidence for association of dopamine D2 receptor variant (Ser311/Cys311) with major psychosis. Am. J. Med. Genet. 67, 415-417 (1996). 
13 Arinami, T., Itokawa, M., Enguchi, H., Tagaya, H., Yano, S., Shimizu, H. et al. Association of dopamine D2 receptor molecular variant with schizophrenia. Lancet 343, 703-704 (1994).

14 Arinami, T., Itokawa, M., Aoki, J., Shibuya, H., Ookubo, Y., Iwawaki, A. et al. Furthe association study on dopamine D2 receptor variant S311C in schizophrenia and affective disorders. Am. J. Med. Genet. 67, 133-138 (1996).

15 Serretti, A., Macciardi, F. \& Smeraldi, E. Dopamine receptor D2 Ser/Cys311 variant associated with disorganized symptomatology of schizophrenia. Schizophr. Res. 34, 207-210 (1998).

16 Serretti, A., Lattuada, E., Lorenzi, C., Lilli, R. \& Smeraldi, E. Dopamine receptor D2 Ser/Cys 311 variant is associated with delusion and disorganization symptomatology in major psychoses. Mol. Psychiatry. 5, 270-274 (2000).

17 Rybakowski, J. K., Borkowska, A., Czerski, P. M., Kapelski, P., Dmitrzak-Weglarz, M. \& Hauser, J. An association study of dopamine receptors polymorphisms and the Wisconsin card sorting test in schizophrenia. J. Neural. Transm. 112, 1575-1582 (2005).
18 Xu, H., Kellendonk, C. B., Simpson, E. H., Keilp, J. G., Bruder, G. E., Polan, H. J. et al DRD2 C957T polymorphism interacts with the COMT Val158Met polymorphism in human working memory ability. Schizophr. Res. 90, 104-107 (2007).

19 Markett, S. A., Montag, C. \& Reuter, M. The association between dopamine DRD2 polymorphisms and working memory capacity is modulated by a functional polymorphism on the nicotinic receptor gene CHRNA4. J. Cogn. Neurosci. 22, 1944-1954 (2010).

20 Hirvonen, J., van Erp, T. G., Huttunen, J., Nagren, K., Huttunen, M., Aalto, S. et al. Striatal dopamine D1 and D2 receptor balance in twins at increased genetic risk for schizophrenia. Psychiatry Res. 146, 13-20 (2006).

21 Hanninen, K., Katila, H., Kampman, O., Anttila, S., Illi, A., Rontu, R. et al. Association between the C957T polymorphism of the dopamine D2 receptor gene and schizophrenia. Neurosci. Lett. 407, 195-198 (2006).

22 Lawford, B. R., Young, R. M., Swagell, C. D., Barnes, M., Burton, S. C., Ward, W. K. et al. The C/C genotype of the C957T polymorphism of the dopamine D2 receptor is associated with schizophrenia. Schizophr. Res. 73, 31-37 (2005) 